\title{
Management of Orbital Floor Fractures: An Oculoplastic Surgeon's View
}

${ }^{1}$ Surbhi Arora, ${ }^{2}$ Ashok Kumar Grover, ${ }^{3}$ Shaloo Bageja

\begin{abstract}
Orbital fractures commonly occur in maxillofacial trauma causing enophthalmos and diplopia. The isolated orbital floor fracture often called as blow out fracture is a common presentation following blunt trauma. The floor fractures may also occur in combination with zygomatic arch fractures, Le Fort II or III or with fracture of other orbital bones. The cosmetic and functional problems can virtually become untreatable if floor fractures are not properly managed. In recent years, management of these fractures has been standardized with special emphasis on methods of treatment, timing and materials used for reduction.
\end{abstract}

Keywords: Blowout fractures, Diplopia, Enophthalmos, CT scan, Swinging eyelid approach.

How to cite this article: Arora S, Grover AK, Bageja S. Management of Orbital Floor Fractures: An Oculoplastic Surgeon's View. J Postgrad Med Edu Res 2014;48(2):75-80.

\section{Source of support: Nil}

Conflict of interest: None

\section{INTRODUCTION}

The bony orbit and its soft tissues are frequently involved in facial trauma occasionally resulting in orbital floor fractures. The isolated orbital floor fracture often called as blow out fracture is a common presentation following blunt trauma. The most common orbital walls affected are floor and medial wall. The floor fractures may also occur in combination with zygomatic arch fractures, Le Fort II or III or with fracture of other orbital bones. However, isolated orbital floor fracture represents $21.4 \%$ of all facial fractures. ${ }^{1}$

The alternative buckling force theory proposed by Fujino ${ }^{4}$ suggests that when infraorbital rim is displaced posteriorly, the force is transmitted to the orbital floor which buckles and fractures while the rim returns to its normal position without fracturing. This concept was supported by a experimental model.

\footnotetext{
${ }^{1}$ Senior Resident, ${ }^{2}$ Director, ${ }^{3}$ Consultant

${ }^{1}$ Department of Ophthalmology, Vision Eye Centre, New Delhi, India

${ }^{2,3}$ Department of Oculoplasty, Vision Eye Centre, New Delhi India
}

Corresponding Author: Surbhi Arora, Senior Resident Department of Ophthalmology, Vision Eye Centre, New Delhi India, Phone: 8447327088, e-mail: arorasurbhi1981@gmail.com
The two theories proposed for the development of blow out fracture are 'hydraulic' and 'buckling'. According to Smith and Regan ${ }^{2}$ older hydraulic theory (Fig. 1), when an object larger than the orbital entrance impacts the globe and the upper eyelid, it transmits the kinetic energy to the periocular tissue. ${ }^{3}$ To relieve the increased intraorbital pressure, the floor blows out into the maxillary sinus. Most fractures occur in the posteromedial region of the orbit that is comprised of the thinnest bone.

A comprehensive assessment of associated ocular injuries and injuries to other parts of the body is required in a patient presenting with orbital trauma. The possibility of a globe rupture should always be ruled out. A globe rupture should be treated first and then an orbital fracture should be diagnosed and managed. Assessment of pupil is essential as pupillary dysfunction along with decreased visual acuity, may be due to traumatic optic neuropathy. Forced duction test is very essential.

Clinical features of a patient with orbital floor fracture may vary according to the time of presentation. Common features include eyelid ecchymosis/hematoma (Figs 2A and B), subcutaneous emphysema, infraorbital hypoesthesia, restriction of ocular motility with diplopia (Fig. 3), initial proptosis followed by enophthalmos (Figs 4A and B).

Suspicion of an orbital floor fracture is based on clinical features and is usually confirmed radiologically.

The patient can be investigated by radiological means, such as X-rays, CT and MRI scans.

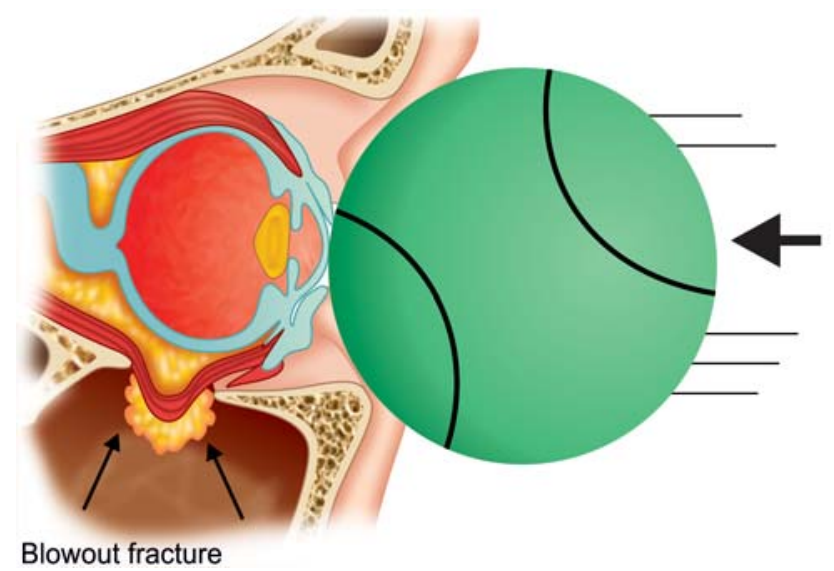

Fig. 1: Hydraulic theory of blowout fracture 
$\mathrm{X}$-ray has limited usefulness due to lack of ability to detect differences of less than $10 \%$ in tissue densities.

The gold standard modality for evaluation of orbital fractures is CT scan (Figs 5A and B). CT scan without contrast provides views of high density bone. The tear drop sign is the characteristic of orbital fracture on CT scan. Both axial and coronal 1.5 to $2.00 \mathrm{~mm}$ cuts should be ordered to properly evaluate the orbit and the floor. It has the advantage of delineating soft tissue of the eye, orbit and adnexae. Spiral CT may be used in the acutely injured patient because of its rapid scan technique, providing smooth data for the three dimensional reformations, and may demonstrate foreign bodies in more than one plane. ${ }^{5}$

An MRI scan is not the first line diagnostic modality. However, it can be done in cases of suspected intracranial injuries or optic nerve pathology.

Indications and timing of repair of orbital fractures are debatable. Not all fractures need to be repaired. Whenever in doubt the patient needs to be re-evaluated every 2 to 3 days during the first 2 weeks. Most fractures can be repaired within 2 weeks except for white eyed fractures which need immediate repair. Orbital hemorrhage and edema will resolve rapidly over the first week. Computed tomography has become a widely accepted and reliable adjunct to help to determine the need for early surgical intervention.
The goal of treatment is reduction of the fracture fragments to restore the orbital anatomy and to restore the physiologic functions and the esthetic appearance of an individual. The incarcerated and depressed contents are released and areas of defect are bridged with supports and implants. Recent advances have made it possible to diagnose early and manage orbital fracture and prevent its troublesome sequelae.

Currently, the indications for the repair of the fracture of orbit have been divided into three groups: immediate repair, repair within 2 weeks and observation.

\section{Immediate Repair ${ }^{6}$}

1. Nonresolving oculocardiac reflex with entrapment causing nausea, vomiting, syncope, bradycardia and heart block.

2. Early enophthalmos or hypoglobus causing facial asymmetry.

3. 'White eyed' floor fracture with entrapment (Figs 6 and 7).

\section{Repair within 2 Weeks}

1. Symptomatic diplopia with positive forced duction test (Fig. 8).

2. Large fracture causing enophthalmos (Figs 9 to 11).

3. Significant hypoglobus (see Figs 9 and 10).

4. Progressive infraorbital hypoanesthesia.

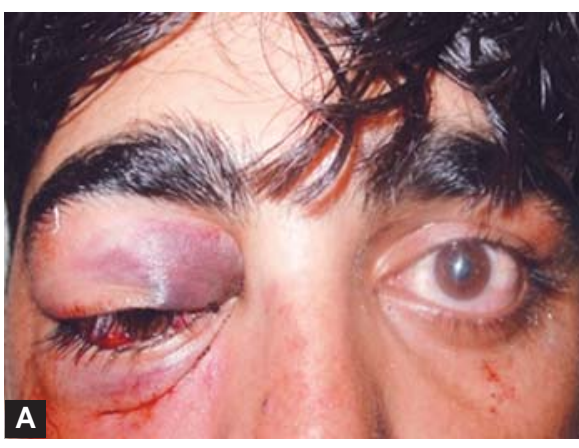

Figs 2A and B: Ecchymosis

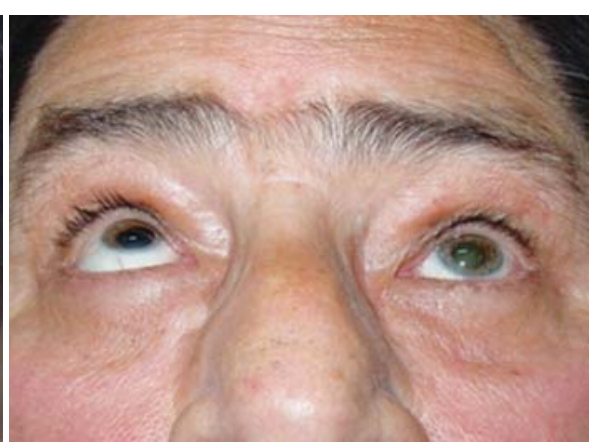

Fig. 3: Restriction of upward/downward gaze/diplopia

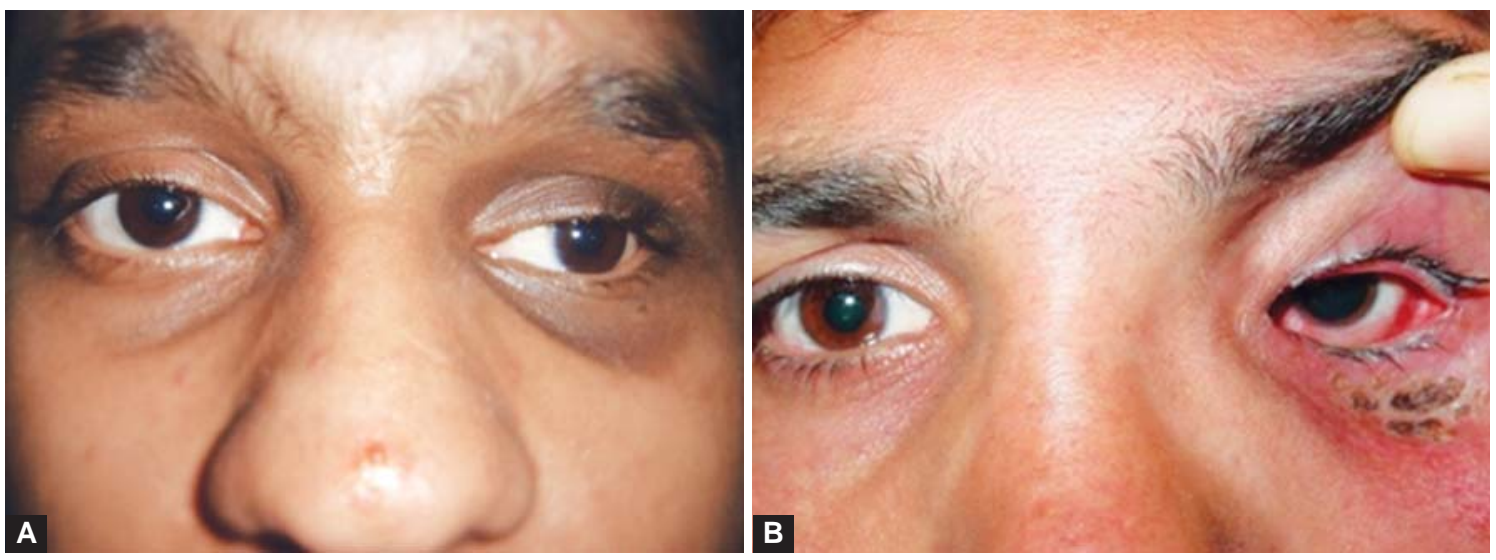

Figs 4A and B: Enophthalmos and hypophthalmos 


\section{Observation}

1. Minimal diplopia but not present in the primary gaze.

2. Good ocular motility.

3. No significant enophthalmos.

4. No significant hypoglobus.

\section{Surgical Management}

The surgical approach is the same, whether performed immediately or within 2 weeks. The repair done during the first 2 weeks offers good chances of recovery for the restoration of ocular motility. Beyond this period, adhesions between the bone fragments and the orbital soft tissue may develop and render the repair more difficult. A residual diplopia may persist because of fibrotic changes.

In the preoperative phase, the patient is advised to avoid blowing the nose to prevent orbital emphysema. Prophylactic antibiotic, antihistamine and decongestants are prescribed.
Some surgeons advocate initial high dose steroids to reduce inflammation. During the preoperative phase an estimation of extent of the fracture as well as of enophthalmos helps surgeon to plan the shape and size of the implant to correct the deformity.

The orbital floor can be repaired through following three approaches:

1. Transconjunctival incision (Table 1 and Flow Chart 1).

2. Transcutaneous-subciliary or over the inferior orbital rim incision (Table 2 and Flow Chart 2).

3. Caldwell-Luc approach.

The transconjunctival incision approach is most preferred one, however, due to lack of access it is combined with lateral canthotomy and cantholysis (swinging eyelid approach). Some surgeons prefer subciliary transcutaneous incision. The only drawback of this approach is that postoperatively patients may develop ectropion or inferior sclera show (Figs 12 to 17$)$.

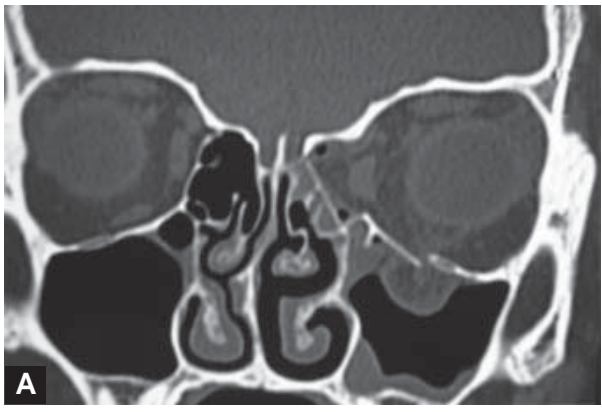

Figs 5A and B: Coronal CT scan depicting floor fracture with herniation of orbital content

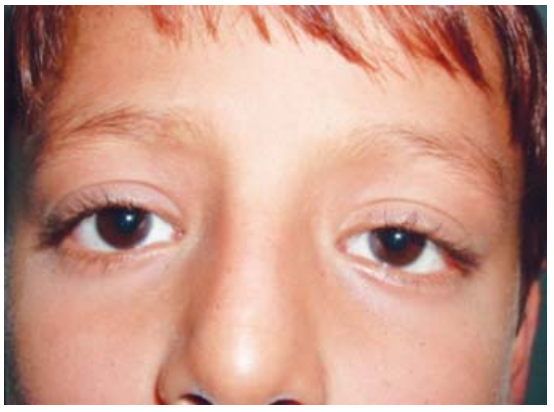

Fig. 6: White eyed fracture

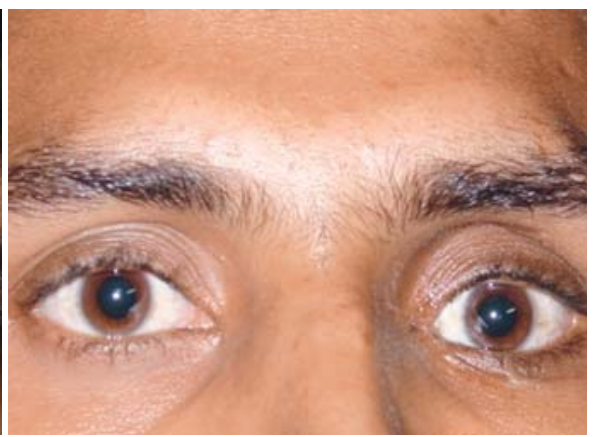

Fig. 9: Enophthalmos/hypoglobus
Fig. 7: Incarcerated contents on CT scan

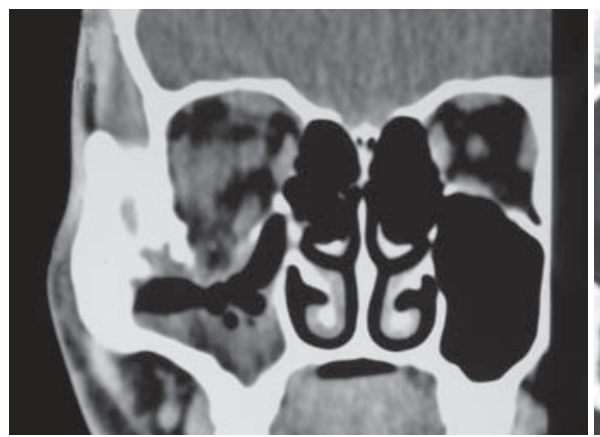

Fig. 10: Coronal CT scan depicting orbital floor fracture

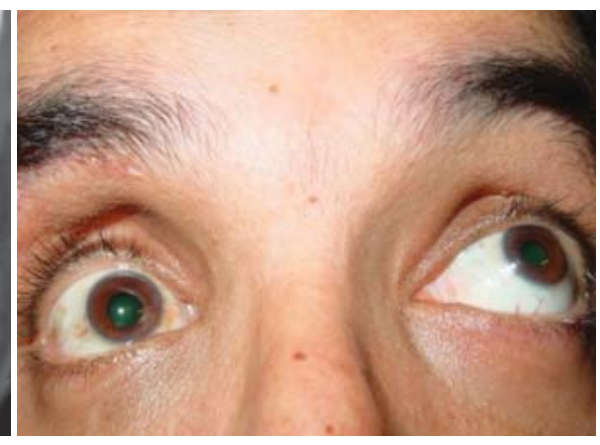

Fig. 8: Diplopia with positive forced duction test

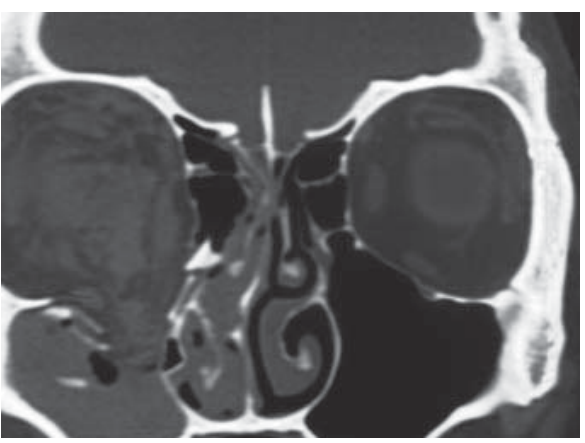

Fig. 11: Large fractures with large herniation of orbital contents

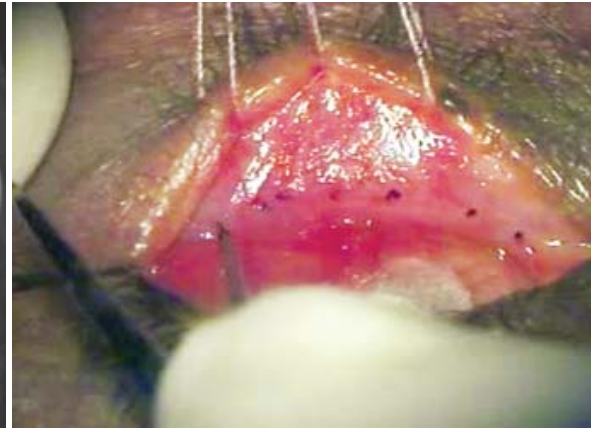

Fig. 12: Traction sutures and incision given 
Flow Chart 1: Transconjunctival approach ${ }^{7}$ swinging eyelid approach

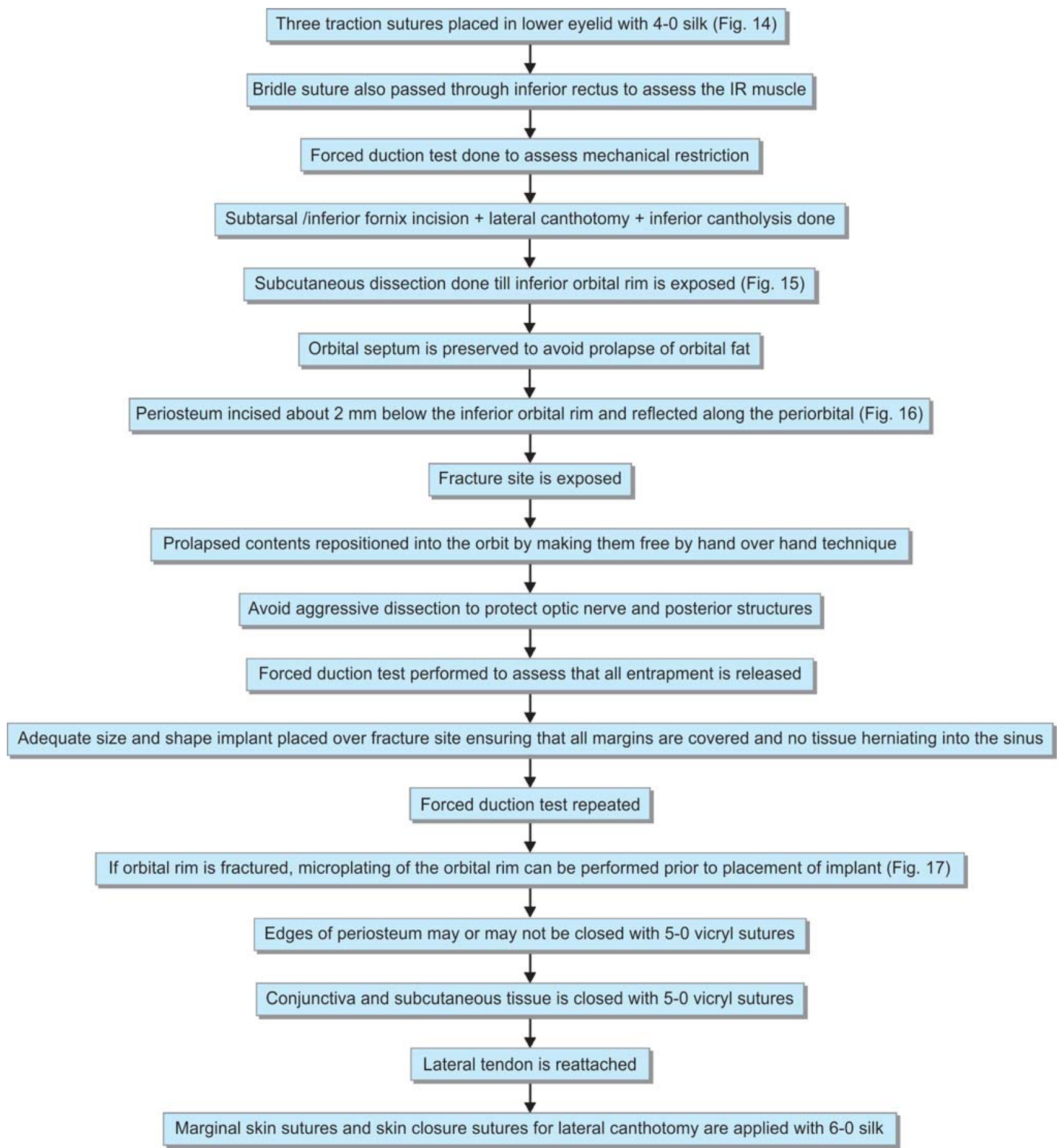

Table 1: Ralph E Wesley stated that the transconjunctival approach $^{7}$ provides a entire wide access to orbit without surface scars as compared to limitation of producing a noticeable scar associated with lower lid retraction that is often permanent and generally caused by skin approaches ${ }^{7}$

\begin{tabular}{l} 
Conjunctival approach \\
\hline Advantage \\
Better cosmesis \\
Disadvantages \\
Comparatively limited exposure \\
Corneal abrasions \\
Hematoma formation \\
Symblepharon formation
\end{tabular}

Surgery can be performed under general anesthesia or local anesthesia with sedation. Local anesthetic $2 \%$ xylocaine with adrenaline 1:100000 with $0.5 \%$ bupivacaine is infiltrated along the inferior orbital rim and lateral canthus.

\section{CALDWELL-LUC APPROACH}

This approach is rarely used. In this, an opening is made into the maxillary sinus in the area of the superior gingiva.

\section{ORBITAL IMPLANT MATERIALS}

Both alloplastic (Table 3 and Fig. 18) and biological (autologous/homologous) (Table 4) materials have been used. The 
characteristics of an ideal alloplastic implant material are that it is of correct size, sterilizable, have adequate strength and possess physical, chemical and biological inertness. It must be durable, non-carcinogenic, stable, easily manipulable and allows single stage reconstruction.

Flow Chart 2: Transcutaneous approach

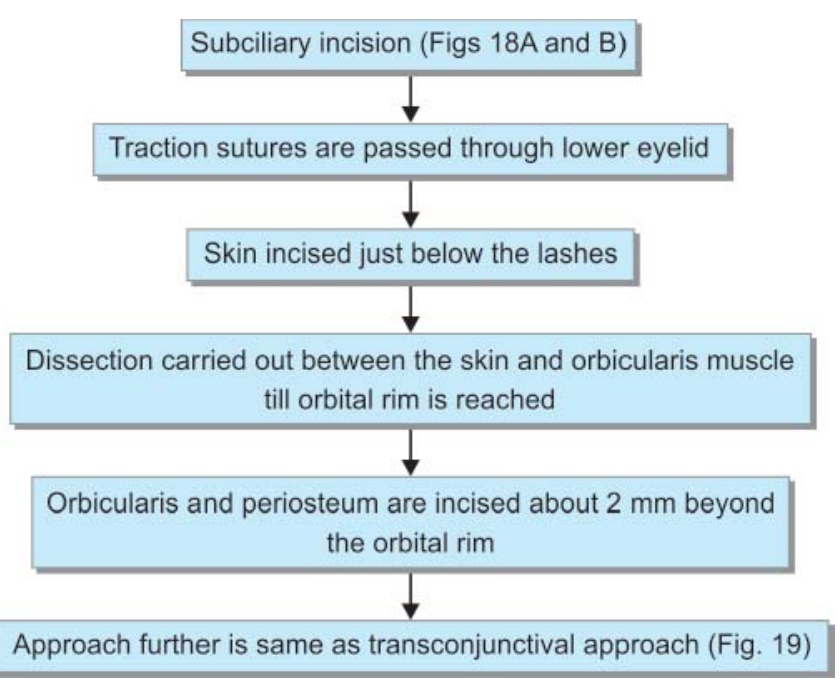

Table 2: Subciliary approach

\begin{tabular}{l}
\hline Subciliary approach \\
\hline Advantages \\
Easy to perform \\
Gives wide access to floor of orbit \\
Disadvantages \\
Visible scarring \\
Ectropion \\
Lower lid retraction \\
\hline
\end{tabular}

Table 3: Alloplastic materials

\begin{tabular}{ll}
\hline Absorbable & Nonabsorbable \\
\hline Polymerized polyactide & E-PTFE \\
Gelatin film & Silastic implants \\
Polyglactin mesh & Teflon implants \\
& Metallic alloys \\
& Hydroxy apatite \\
\hline
\end{tabular}

Table 4: Biological material

Bone graft from iliac crest and ribs

Autologous or banked fascia lata

Irradiated coastal cartilage and calvarium

\section{Postoperative Complications}

They can be minimized by good surgical technique. Some of the complications are:

1. Loss of vision.

2. Persistence of ocular motility restriction and diplopia.

3. Lower eyelid retraction.

4. Infraorbital nerve damage.

5. Persistence of enophthalmos.

6. Symblepharon.

7. Implant extrusion.

8. Orbital infection.

Loss of vision is a rare but dreaded complication. It may be caused by orbital hemorrhage or due to compression of the optic nerve by malpositioning or placement of a large implant. Adequate hemostasis should be achieved before closure. Patients visual acuity should be monitored in the recovery room in the early postoperative period. The orbital implant should be of appropriate size and shape and should not be pushed posteriorly.

Patients should be explained that the diplopia (Figs 19A and B) may get worse in the early postoperative period. It usually resolves over a period of 4 to 6 weeks postoperatively. Force duction test should be performed after the placement of the implant to assess any residual tissue entrapment as well as the motility of the globe.

Lower eyelid retraction (Fig. 20) is most commonly seen with the subciliary incision. It is either due to incorrect closure of periosteum with inclusion of the orbital septum within the sutures or due to the adhesions of the orbital septum to the infraorbital rim. This can be prevented by meticulous dissection between the orbicularis and orbital septum and proper closure.

Patient should be explained about the permanent sensory loss in the distribution of the infraorbital nerve or it may take several months for its recovery.

Residual enophthalmos (Figs 21A and B) is either due to placement of smaller implant or due to failure to treat medial wall fractures. It may also occur due to delayed fat atrophy. It gradually improves over time.

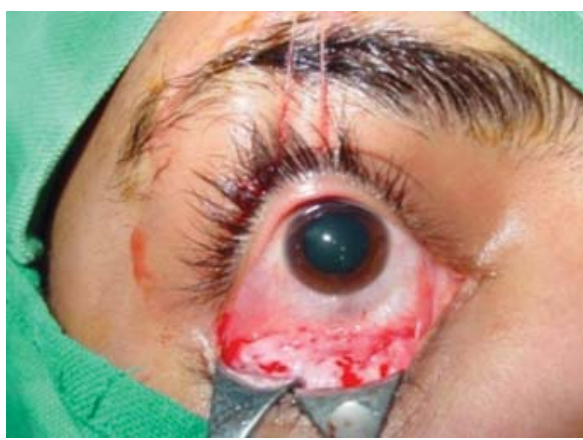

Fig. 13: Subcutaneous dissection done

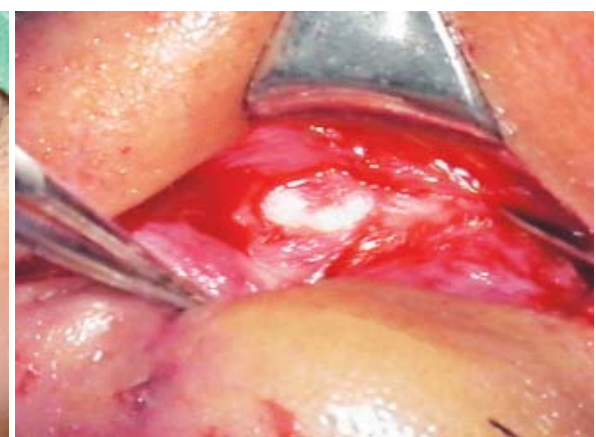

Fig. 14: Periosteal dissection

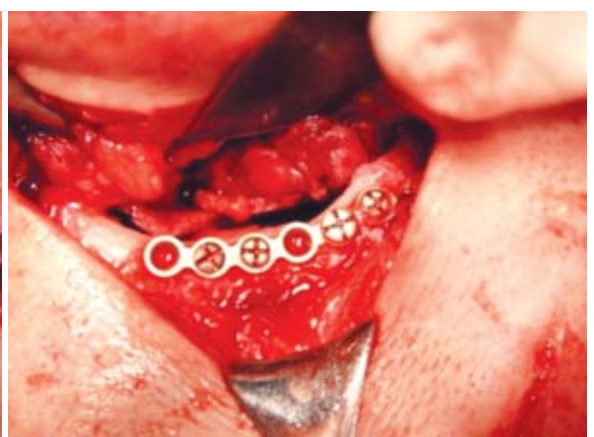

Fig. 15: Microplating of orbital rim 


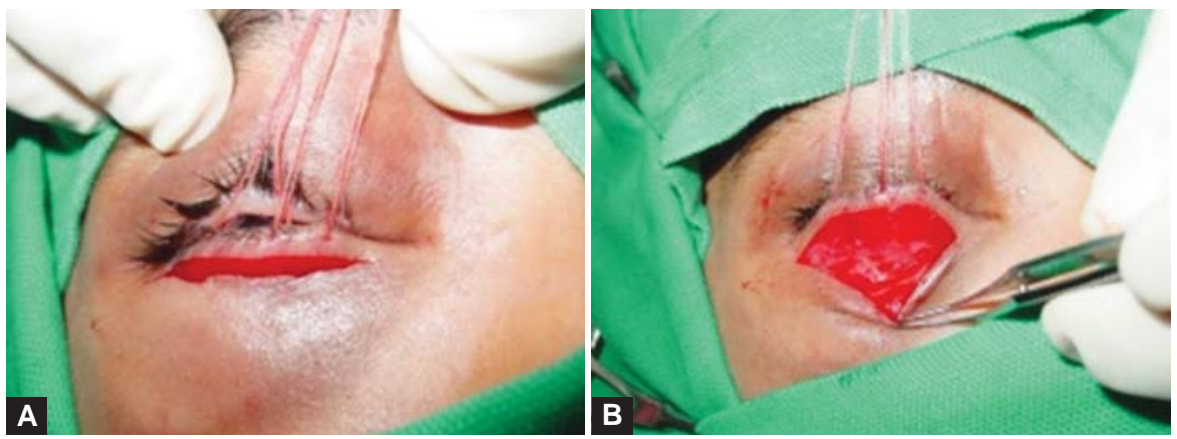

Figs 16A and B: Subciliary incision

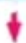

$\downarrow$

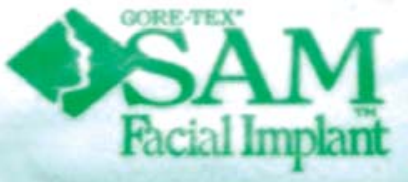

Fig. 18: E-PTFE implant

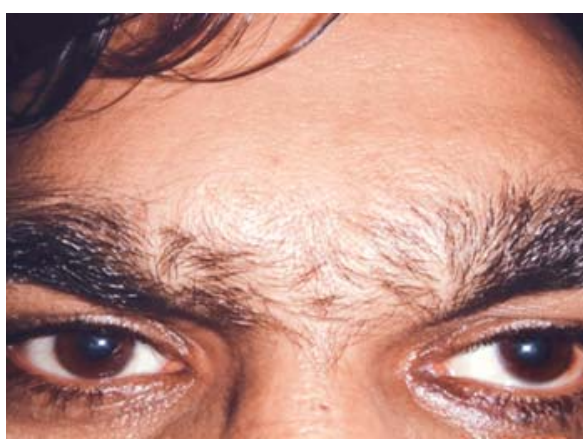

Fig. 20: Lower eyelid retract

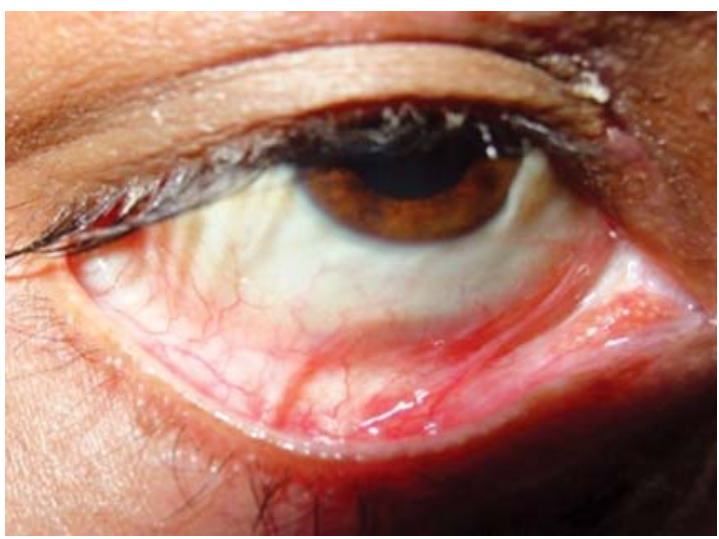

Fig. 22: Symblepharon

Symblepharon (Fig. 22) is seen following a transconjunctival incision, usually more common with forniceal incision than subtarsal incision.

The extrusion of implant may occur either due to infection, oversized implant or inadequate closure of the periosteum to the inferior orbital rim.

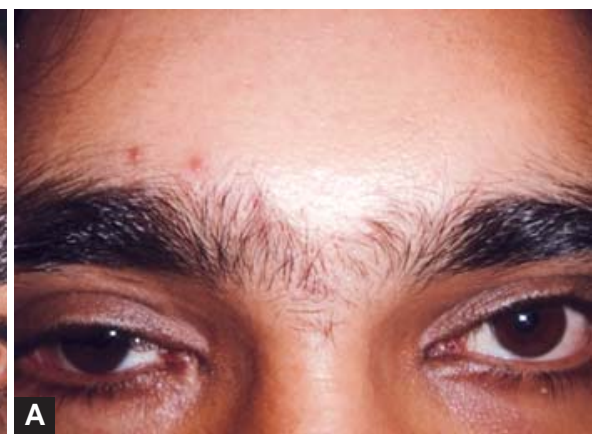

\section{REFERENCES} 1989;29:388. 241-252.

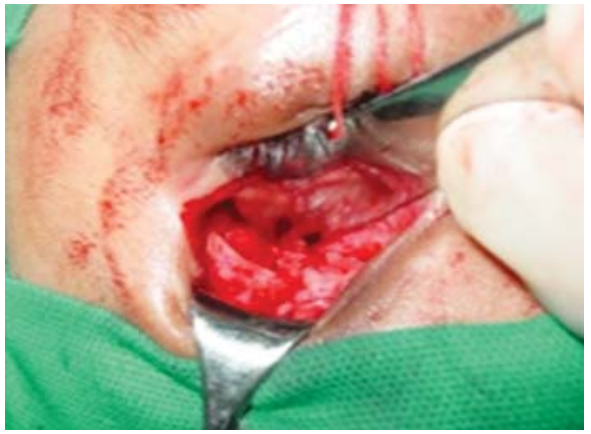

Fig. 17: E-PTFE implant put

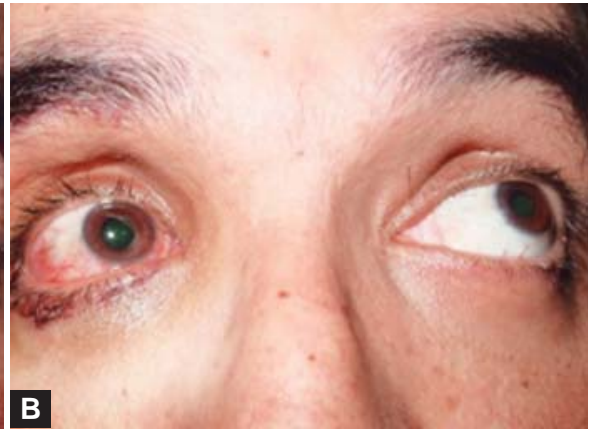

Figs 19A and B: Diplopia and restriction of ocular motility

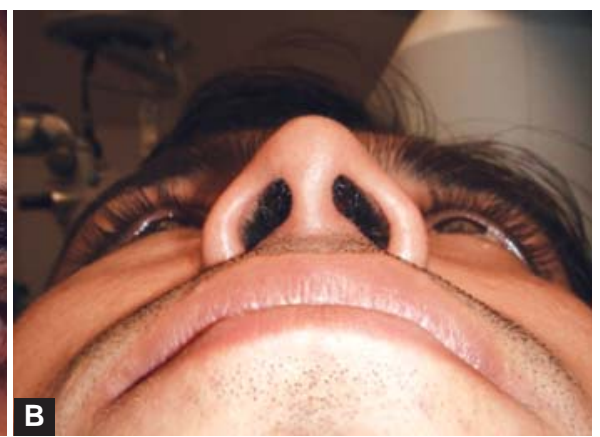

Figs 21A and B: Residual enophthalmos

Orbital infection is managed by intravenous antibiotics but may sometimes require explantation of the implant.

1. Seberer M, Sullivan WG, Smith DJ Jr. An analysis of 1423 Facial fractures in 788 patients at an urban trauma centre. J Trauma

2. Smith B, Regan WF. Blow-out fractures of the orbit. Smith B, Regan WF. Mechanism and correction of internal orbital fracture. Am J Ophthalmol 1957 Dec;44(6):733-739.

3. Emery JM, Van Noorden GK, Schlernitzaver DA. Management of orbital floor fracture. Am J Ophthalmol 1972;74:299-306.

4. Courtney DJ, Thomas S. Isolated orbital blow out fractures: survey and review. Br J Oral Maxillofac Surg 2000;496-502.

5. Mauriello JA, Lee HJ, Nguyen L. CT of soft tissue injury and orbital fractures. Radiol Clin North Am 1999 Jan;37(1):

6. Burnstine MA. Clinical recommendations for repair of orbital facial fractures. Curr Opin Ophthalmol 2003;14(5):236-240.

7. Wesley RE. Transconjunctival approaches to the lower lid and orbit. J Oral Maxillofac Surg 1998;56:66-69. 